\title{
Vibration on board and health effects
}

\author{
Anker Jensen $^{1}$, Jørgen Riis Jepsen ${ }^{1,2}$ \\ ${ }^{1}$ Department of Occupational Medicine, Hospital of South Western Jutland, Esbjerg, Denmark \\ ${ }^{2}$ Centre of Maritime Health and Society, Institute of Public Health, University of Southern Denmark, Esbjerg, Denmark
}

\begin{abstract}
There is only limited knowledge of the exposure to vibrations of ships' crews and their risk of vibration-induced health effects. Exposure to hand-arm vibrations from the use of vibrating tools at sea does not differ from that in the land-based trades. However, in contrast to most other work places, seafarers are also exposed to vibrations to the feet when standing on vibrating surfaces onboard. Anecdotal reports have related the development of "white feet" to local exposure to vibration, e.g. in mining, but this connection has not been investigated in the maritime setting. As known from studies of the health consequences of whole body vibrations in land-transportation, such exposure at sea may affect ships' passengers and crews. While the relation of back disorders to high levels of whole body vibration has been demonstrated among e.g. tractor drivers, there are no reported epidemiological evidence for such relation among seafarers except for fishermen, who, however, are also exposed to additional recognised physical risk factors at work. The assessment and reduction of vibrations by naval architects relates to technical implications of this impact for the ships' construction, but has limited value for the estimation of health risks because they express the vibration intensity differently that it is done in a medical context.
\end{abstract}

(Int Marit Health 2014; 64, 2: 58-60)

Key words: hand-arm vibration, whole body vibration, ships, maritime, seafarers

\section{INTRODUCTION}

Vibrations are oscillating movements transmitted through solid material. Transmission to structures of the human body may induce health effects, i.e. on the musculoskeletal system, either directly or indirectly through the impact of reflex muscle activity. In addition to the health effects on humans, vibrations on ships may affect the durability of the mechanical structure of the ship and thus are of technical interest as well.

The magnitude of vibrations can be expressed in various ways. In a medical context, the intensity is usually expressed by acceleration $\left(\mathrm{m} / \mathrm{s}^{2}\right)$. Shear forces through acceleration/deceleration is a possible mechanism for the development of tissue damage. Engineers, on the other hand, usually express vibrations as velocity or displacement. The assessment of vibrations is further complicated since their frequency and direction also have to be considered. The perception of vibrations and their impact on the tissues depend of their frequency, which has to be weighted differently for the assessment of the dose. The acceleration may occur in all six directions, 3 linear and 3 rotational, out of which the 3 linear directions, vertical (Z-axis), transversal (Y-axis) and longitudinal (X-axis) are regarded as the most important. Consequently, the standards for measuring vibrations [1, 2] only take these directions in account. The overall vibration exposure can be calculated through the root-mean-square method.

Exposure limits for humans and action values, above which measures to reduce exposure should be applied, are established internationally and within the European Union [3], with standards for assessing vibration exposure, including frequency weighting.

Vibrations are of special concern in the transport trades. Both in land and air transport and at sea the propulsion system produces vibrations. Furthermore, uneven surfaces induce vibrations during the movement of vehicles. This is

Anker Jensen, MD, Department of Occupational Medicine, Hospital of South Western Jutland, Esbjerg, Denmark, e-mail: anker.jensen@dadlnet.dk

Jørgen Riis Jepsen, MD, Centre of Maritime Health and Society, Institute of Public Health, University of Southern Denmark, Esbjerg, Denmark, e-mail: jriis@cmss.sdu.dk

NIVA COURSE, MARITIME OCCUPATIONAL MEDICINE, EXPOSURES AND HEALTH EFFECTS AT SEA, $12^{\text {TH }}-15^{\text {TH }}$ MAY 2014, HOTEL MARIENLYST, HELSINGØR, DENMARK 
also the case in ships when waves result in moving the ship hull in various directions. Most vehicles, including ships, are relative lightweight and rigid structures and consequently good transmitters of vibration. Therefore, crew and passengers will inevitably be exposed to vibrations.

Unfortunately the assessment of vibrations for technical purposes may have limited value in a medical context because of different ways of expressing their magnitude, and diversities with regard to priorities concerning measuring points and safety limits. Consequently, a safe level from an engineering point of view may not be safe for humans. It seems that for frequencies above $5-15 \mathrm{~Hz}$, higher vibration levels can be accepted for mechanical structures than for human exposure $[3,4]$.

There is clear evidence for adverse health effects of vibrations in humans. Possible positive effects in physiotherapy are also reported, but will not be considered here. Usually, vibrations from vibrating tools transmitted through the hand causing local tissue damage (hand-arm vibration syndrome - HAVS) and whole-body vibrations (WBV) are considered separately because their health effects differ. On ships, local health effects to the lower extremity through foot-transmitted vibrations could be an issue as well.

\section{LOCAL HEALTH EFFECTS}

Vascular symptoms after prolonged exposure to vibrating hand tools have been known since nearly 100 years [5]. The association between exposure to hand-arm vibrations and both vascular and neurological disorders causing white fingers and tingling and numbness of the fingers has been demonstrated in several studies [6]. Based on epidemiological data a dose-response relation has been established [1], although some controversy remains on this issue concerning the frequency weighting [5]. The prevalence of HAVS after prolonged exposure may exceed $50 \%$ in some risk professions [5]. Especially white fingers might be of special concern in maritime medicine because symptoms are likely to occur more often in the cold and wet conditions that often prevail at sea. Otherwise, the exposure to vibrations through hand-held tools such as grinders is not specific to job tasks on board ships but is comparable to similar exposures in land-based trades.

Further potential health risks after exposure to handarm vibration are neuropathic conditions such as carpal tunnel syndrome, osteoarthritis and muscle weakness [1]. A hospital registry study has demonstrated a significantly elevated relative risk for carpal tunnel syndrome for fishermen (SIR 267) and for non-officers at sea (SIR 180) [7], but this finding could also be explained by exposures other than vibrations such as heavy pulling and lifting. Specific epidemiological data on HAVS in seamen is scarce.

Crew members on ships, primarily engine-men, are also exposed to vibrations transmitted to the feet while standing on vibrating surfaces and consequently experience health effects on the lower extremity analogue to upper limb HAVS. The generally accepted magnitude of vibrations of surfaces on ships from a technical point of view [4] seems to be well above the magnitude that is prone to induce HAVS for frequencies above 10-15 Hz. Consequently, health effects on the lower extremity after prolonged standing on vibrating surfaces on ships might be a possible outcome. Health effects to the lower extremity ("white-feet") related to standing on vibrating surfaces have been reported in i.e. mining [8], but similar cases have not been reported for ship crews. Medically applicable threshold values or specific exposure limits to the feet have not been established.

The mechanism behind HAVS is not completely understood. Although local as well as central mechanisms have been proposed, local mechanisms are currently regarded as playing the major role [9]. Therefore symptoms in the feet should not be expected after exposure to vibration of the hands and vice versa.

\section{WHOLE BODY VIBRATIONS}

Both passengers and crews are exposed to WBV in ships, as well as in land vehicles and aircrafts. Vibrations are transmitted to the body through the pelvis in the sedentary position and through the feet in the standing position. As most studies have dealt with WBV in land transport, the evidence on health effects is mainly related to the sedentary position. Furthermore, the focus has been on a daily exposure of up to 8 hours, reflecting the normal workday. In contrast, a 24-hour exposure is the rule on board ships, although the exposure levels might differ during the day.

Frequencies between 1 and $80 \mathrm{~Hz}$ are considered as relevant for "health, comfort and perception" and frequencies below $0.5 \mathrm{~Hz}$ are considered as relevant in connection with motion sickness [2].

The standard for the assessment of the magnitude of WBV [2] has established caution zones concerning health effects despite limited data reflecting dose-response relations. The caution zones are, at least partly, based on observations relating to comfort and discomfort [10]. A special standard for vibration exposure on ships [11] has been established as well as acceptance criteria for ship vibration in order to avoid damage on local technical structures [4]. The latter is below the limits for human exposure for low frequencies $(<7-15 \mathrm{~Hz})$ but at higher frequencies the technical structures seems to endure more than human tissues. Measuring vibrations under standard conditions may not necessarily reflect the actual exposure, which is also highly depending on variable conditions, such as weather and sea in combination with the type of ship. This may primarily influence the very low frequencies.

There is strong epidemiological evidence that exposure to WBV is a risk factor for health effects affecting primarily 
the spine. Several epidemiological studies have demonstrated a link between WBV and low back disorders although the dose-response relationship is not quite clear [12]. Studies on drivers of land-based vehicles, and operators of construction and forestry machinery have shown a prevalence odds ratio of up to 3.3 for low back pain [13], which is regarded as likely related to their WBV exposure. One study [14] showed a significantly elevated risk for disability pension among workers exposed to WBV in general (HR 1.61). Epidemiological studies on this relationship among seamen and fishermen are scarce. A significantly elevated risk for thoraco-lumbar disorders (ICD M51) has been demonstrated among fishermen (SIR 185), but not for seamen (officers and non-officers) [7]. Fishermen are also generally more exposed to strenuous work tasks, which must usually be regarded as their primary risk factor for spinal disorders.

Even though epidemiological studies show an elevated risk of back disorders for workers exposed to WBV, it is not possible to demonstrate any specific changes in the spine related to WBV [15]. Although the mechanism of low back pain is not well understood, it is assumed that axial, shear and torsion forces on the spine and muscular strain play a role. These factors are interconnected. The muscular arrangement leads to a considerably increased load on the spine through muscular activity. Accelerations, such as through movements of a ship at sea, can be expected to increase the need for stabilising the spine or for compensating through muscular activity. This leads to an increased muscle strain and axial load of the spine [16]. A reduced oxygenation of the spinal muscles related to WBV exposure has been demonstrated [17]. In any event, epidemiology does not indicate, that vibrations related to staying on board a ship as such leads to an increased risk of back disorders.

The resonance behaviour of different organs has been considered relevant in connection with functional symptoms and discomfort during exposure to WBV and has influenced the frequency weighting. For thoracic and abdominal organs the resonance frequency is $3-9 \mathrm{~Hz}$, and WBV exposure in these frequency bands can cause abdominal discomfort and chest pain. Visual disturbances at frequencies around $60-90 \mathrm{~Hz}$ is probably due to resonance of the eyeball [18]. A dose-response relation between WBV and discomfort has been established [2], but may not necessarily reflect a reliable indication of a dose-response relation for long term health effects.

\section{REFERENCES}

1. ISO 5349, Mechanical vibration - measurement and evaluation of human exposure to hand-transmitted vibration, Part 1, 2001.

2. ISO 2631, Mechanical vibration and shock - evaluation of human exposure to whole body vibration, Part 1, 1997.

3. EU Directive 2002/44/EC on the minimum health and safety requirements regarding the exposure of workers to the risks arising from physical agents (vibration), Luxembourg, 2002.

4. Guidance notes on ship vibration, American Bureau of Shipping (ABS), Houston, 2006.

5. Fridén J. Vibration damage to the hand: clinical presentation, prognosis and length and severity of vibration required. J Hand Surg Br 2001; 26: 471-474.

6. Bovenzi M. Epidemiological Evidence for new frequency weighting of hand-transmitted vibration. Ind Health 2012; 50: 377-387.

7. Kaerlev L, Jensen A, Nielsen PS, Olsen J, Hannerz H, Tüchsen F. Hospital contacts for injuries and musculoskeletal diseases among seamen and fishermen: a population-based cohort study. BMC Musculoskelet Disord 2008; 9: 8.

8. Eger T, Thompson A, Leduc $\mathrm{M}$ et al. Vibration induced white-feet: overview and field study of vibration exposure and reported symptoms in workers. Work 2014; 47: 101-110.

9. Stoyneva Z, Lyapina M, Tzvedkov D, Vodenicharov E. Current pathophysiological views on vibration-induced Raynaud's phenomenon. Cardiovasc Res 2003; 57: 615-624.

10. Mansfield NJ. Human response to vibration. CRC Press, Boca Raton, 2005

11. ISO 6954, Mechanical vibration - guidelines for the measurement, reporting and evaluation of vibration with regard to habitability on passenger and merchant ships, 2000.

12. Lings $\mathrm{S}$, Leboeuf-Yde $\mathrm{C}$. Whole-body vibration and low back pain: a systematic, critical review of the epidemiological literature 1992-1999. Int Arch Occup Environ Health 2000; 73: 290-297.

13. Bovenzi M, Hulshof CTJ. An updated review of epidemiologic studies on the relationship between exposure to whole-body vibration and low back pain (1986-1997); Int Arch Occup Environ Health 1999; 72: 351-365.

14. Tüchsen $F$, Feveile $H$, Christensen KB, Krause $N$. The impact of self-reported exposure to whole-body-vibrations on the risk of disability pension among men: a 15-year prospective study. BMC Public Health 2010; 10: 305.

15. Bible JE, Choemprayong S, O'Neill KR, Devin CJ, Spengler DM. Whole-body vibration. Is there a causal relationship to specific imaging findings of the spine? Spine 2012; 37: E1348-E1355.

16. Chan SCW, Ferguson SJ, Gantenbein-Ritter B. The effects of dynamic loading on the intervertebral disc. Eur Spine J 2011; 20: 1796-1812.

17. Maikala RV, Bhamphani YN. In vivo lumbar erector spinae oxygenation and blood volume measurements in healthy men during seated whole-body vibration. Exp Physiol 2006; 91: 853-866.

18. Griffin MJ. Handbook of human vibration, Academic Press Ltd, London, 1990. 